\title{
Measuring changes in Schlemm's canal and trabecular meshwork in different accommodation states in myopia children: an observational study
}

\author{
Yan Xiang ${ }^{1} \cdot$ Liugui Chen $^{1} \cdot$ Yin Zhao ${ }^{1} \cdot$ Wei Chen $^{1} \cdot$ Zhiqi Chen $^{1} \cdot$ Shiliang Liu ${ }^{1} \cdot$ Sili Jing $^{1} \cdot$ Anne Manyande $^{2}$. \\ Ping Wang ${ }^{1} \cdot$ Hong Zhang ${ }^{1} \cdot$ Junming Wang ${ }^{1}$
}

Received: 29 October 2018 / Revised: 3 July 2019 / Accepted: 8 July 2019 / Published online: 9 August 2019

(c) The Author(s), under exclusive licence to The Royal College of Ophthalmologists 2019

\begin{abstract}
Purpose The aim of the study was to evaluate changes in size of Schlemm's canal (SC) and trabecular meshwork(TM) in response to accommodation stimuli and cycloplegia states in myopia children.

Methods In total, 34 children were enroled in this study. A $-6.0 \mathrm{D}$ accommodation stimulus was achieved by looking at an optotype through a mirror. Cycloplegia state was induced with $1 \%$ tropicamide. Two states were confirmed by measuring the central lens thickness (CLT), anterior chamber depth and pupil diameter. The size of SC and TM was measured using sweptsource optical coherence tomography. The association between changes in SC size and CLT was analysed.

Results Compared with that in the relaxation state, SC size increased significantly under $-6.0 \mathrm{D}$ accommodation stimuli. SC area (SCA) increased from $6371 \pm 2517 \mu \mathrm{m}^{2}$ to $7824 \pm 2727 \mu \mathrm{m}^{2}$, SC length (SCL) from $249 \pm 10 \mu \mathrm{m}$ to $295 \pm 12 \mu \mathrm{m}$ and SC width (SCW) from $27 \pm 9 \mu \mathrm{m}$ to $31 \pm 8 \mu \mathrm{m}$. Under the cycloplegia state, SCA decreased to $5009 \pm 2028 \mu \mathrm{m}^{2}$; SCL to $212 \pm$ $14 \mu \mathrm{m}$, and SCW to $22 \pm 5 \mu \mathrm{m}$. Changes in SCA $(r=0.35, P=0.0007)$, SCL $(r=0.251, P=0.0172)$ and SCW $(r=0.253$, $P=0.016)$ were significantly correlated with changes in CLT. TM size was not significantly altered compared to that in the relaxation state. TM length (TML) increased from $562 \pm 45 \mu \mathrm{m}$ to $587 \pm 47 \mu \mathrm{m}$ after exposure to $-6.0 \mathrm{D}$ accommodation stimulus.
\end{abstract}

Conclusion SC size enlarged in response to $-6.0 \mathrm{D}$ accommodation stimuli and shrunk under cycloplegia. TM length increased under the accommodation stimulus state.

\section{Introduction}

Glaucoma has a high global incidence and is the leading cause of irreversible blindness worldwide. Elevated intraocular pressure (IOP) is a major risk factor for glaucoma, which appears to result from increased resistance to aqueous humour outflow. Trabecular meshwork (TM) and Schlemm's canal (SC) are key structures involved in pathways of aqueous humour flow $[1,2]$.

Junming Wang

eyedrwjm@163.com

1 Department of Ophthalmology, Tongji hospital, Tongji medical College, Huazhong University of Science and Technology, 430030 Wuhan, Hubei, China

2 School of Human and Social Sciences, University of West London, London, UK
Clinically, surgical or pharmacological treatments targeting SC and TM alleviate elevated IOP [3]. Therefore, SC and TM have been considered promising therapeutic targets for the treatment of glaucoma. Accommodation stimulation promotes aqueous humour outflow and decreases IOP [4-6]. Conversely, paralysis of accommodation by cycloplegia raises aqueous outflow resistance and IOP [7, 8]. The mechanisms underlying these changes are unclear. Most researchers suggest that the mechanical effects of ciliary muscles under different accommodation states mediate structural changes in TM and SC. However, to date, the effects of accommodation on structural changes in SC and TM have not been observed in vivo in humans. Optical coherence tomography (OCT) provides non-invasive cross-sectional imaging techniques of the eye and produces static and dynamic anterior segment images. Myopia is a known risk factor, especially for primary open-angle glaucoma $[9,10]$. The aim of this study was to explore changes in 
Fig. 1 Simulated diagram of experimental set-up and examples of measured items in optical coherence tomography (OCT) image. a A tilted firstsurface mirror with a frame carrying a rotation axis was attached to the OCT machine and used to place the fixation stimuli at different vergences. Measurements were obtained on the right eye of the subject while the left eye looked at an optotype through the mirror. The figure depicts a simulated diagram (left), whole-view of the testing system (middle), and large view of the optotype in the mirror (right). b Simulated diagram of the anterior eye segment accounting for measured items in this study: central lens thickness (CLT), pupil diameter (PD), Schlemm's canal length (SCL), Schlemm's canal width (SCW), trabecular meshwork length (TML), trabecular meshwork width (TMW), ciliary muscle2 (CM2) and 3 (CM3) $\mathrm{mm}$ posterior to the scleral spur, and scleral spur (SS). c OCT image showing measurement methods for CLT and PD. d OCT image showing measurement methods for anterior chamber depth (ACD). e OCT image showing measured part of SC (yellow loop, including SCL and SCW) and TM (green arrow, including TML and TMW). f Image showing measured part of the ciliary muscle (yellow line marks the testing sites of CM2 and $\mathrm{CM} 3$, respectively)
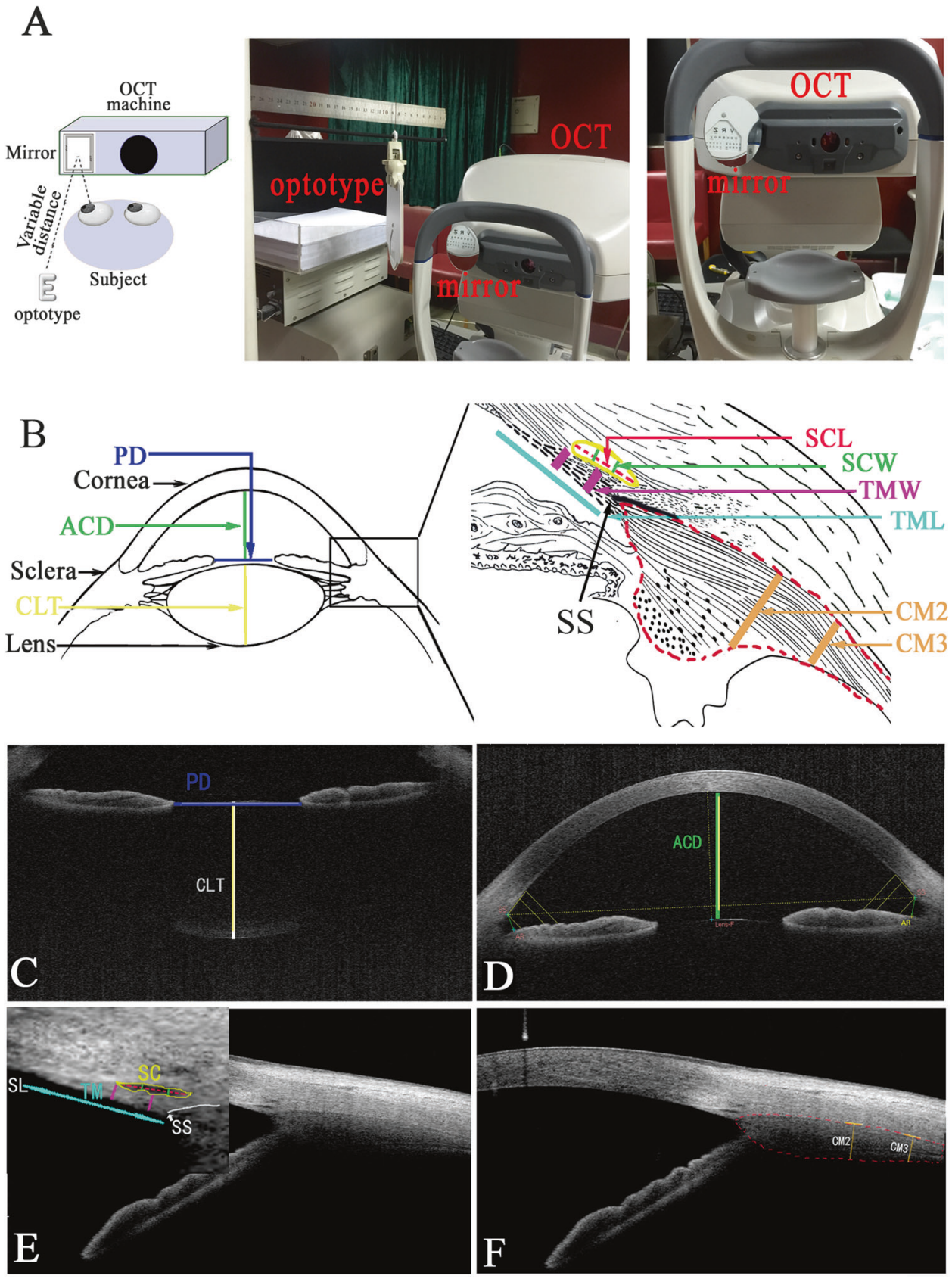

TM and SC structures in different accommodation states (accommodation stimulus, relaxation of accommodation and paralysis of accommodation) in myopia patients by adopting OCT imaging, in order to provide deeper understanding of IOP regulation and glaucoma.

\section{Methods}

Ethics approval was obtained from the local institute's ethics committee (Huazhong University of Science and Technology). The study protocol was registered with chictr.org.cn (ChiCTR-ROC-16008832). Written informed consent was obtained from parents. In total, 34 children at a refraction outpatient clinic of Tongji Hospital were recruited for the study over a period of 4 months between June 2017 and September 2017. Children were aged from 7 to 14 years old, experienced refractive error $(\geq-6.0 \mathrm{D}$ and $\leq-0.5 \mathrm{D}$ with corrected visual acuity of at least 20/20 in Snellen equivalent), and required cycloplegic refraction testing. Exclusion criteria included presence or history of other ocular diseases, amplitude of accommodation $<6.0 \mathrm{D}$, and/or the presence of central nervous system or systemic diseases. 


\section{Experimental procedures}

Serial regular ocular examinations were performed to screen patients with ocular diseases other than refractive error. These included slit lamp, fundus examination, IOP, axial length check and subjective optometry. Subsequently, amplitude of accommodation was measured using minus lens test as reported by León et al. [11]. Patients were excluded if their accommodation amplitude was $<6.0 \mathrm{D}$.

Patients were subjected to an OCT test. The first test was under relaxation state of accommodation, which was achieved by far point staring. Next, subjects were tested under $-6.0 \mathrm{D}$ accommodation stimulation state. This state was achieved by watching mirrored optotypes, which were placed at a distance calculated for each individual based on the formula: $100 /-(-6+X) \mathrm{cm}$, where $X$ was the patient's refractory error value in dioptres. Finally, subjects were tested under the state of cycloplegia. This was achieved by administering $1 \%$ tropicamide eye drops on the corneal surface of patients five times with a 5-min interval in each eye. Measurements were performed $5 \mathrm{~min}$ after the last drops of tropicamide were administered.

\section{Outcome measures: OCT data acquisition and processing}

The primary outcome measure in this study was SC area (SCA) in different accommodation states. Participants underwent examinations of swept-source OCT (CASIA SS-1000; Tomey Corporation, Nagoya, Japan), which is specifically designed for anterior segment imaging using a $1310 \mathrm{~nm}$ wavelength with a scan speed of 30,000 A-scans per second and an axial resolution of $<10 \mu \mathrm{m}$. To enable measurements indifferent states, we employed the procedure reported by Esteve-Taboada [12] by using a tilted first-surface mirror to fix stimuli at different vergences in the left eye. The tilted mirror with a frame carrying a rotation axis was fixed to the OCT machine (see simulated diagram, Fig. 1a). Subjects were instructed to look at optotypes in the mirror with the left eye while measurements were obtained for the right eye. The tilting angle of the mirror was adjusted for every patient according to the particular interpupillary distance, testing items and side. Subjects were instructed to look at an optotype through the tilted mirror, and the optotype was placed at the required distance (far point for the relaxation and cycloplegic states, with an individually calculated distance for the $-6.0 \mathrm{D}$ accommodation state). The angle analysis mode (dimension, a raster of $128 \mathrm{~B}$-scans each with 512 A-scans over $8 \mathrm{~mm}$ ) was used to capture images of the ACD, PD and CLT. Then, the 3D-angle high-definition mode (dimension, a raster of 64 B-scans each with
512 A-scans over $8 \mathrm{~mm}$ ) was selected to capture images of the SC, TM and ciliary muscle at 9 o'clock positions. Conjunctival vessels were used as landmarks to scan the same site under different states. During image acquisition, blinking was not permitted. Each measurement was taken $4 \mathrm{~s}$ later, as the patient's last blink allowed the tear film to spread over the cornea.

\section{Image analysis}

Each image was quantified manually using ImageJ software (http://imagej.nih.gov/ij/; provided in the public domain by the National Institutes of Health, Bethesda, MD, USA). Measured items were determined based on two-dimensional images (for examples of measured items, see Fig. 1b-f). Anterior chamber depth (ACD) was defined as the perpendicular distance between the corneal endothelium at the corneal apex to the anterior lens surface. Central lens thickness (CLT) was defined as the distance between the midpoint of the front and back of the crystal lens. Pupil diameter (PD) was defined as the distance between the edges of the iris. Scleral spur was defined as the point between the TM and ciliary body. Thickness of the ciliary muscle at 2 (CM2) and 3 (CM3) $\mathrm{mm}$ posterior to the scleral spur was assessed (limited by OCT scan depth, we did not analyse data of ciliary muscle width at $1 \mathrm{~mm}$ posterior to the scleral spur). SC area was drawn freehand and depicted the area surrounded by the outline of the SC. SC length was measured from the posterior to anterior SC end point. SC width was calculated by obtaining the two average values of one-third points. TM length was defined as the distance between the scleral spur and Schwalbe's line. According to our previous reports [13, 14], each TM width measurement was performed perpendicular to the inner layer of the meshwork. TM width was calculated as the average of two measurements made at the anterior end point of SC and halfway down the SC.

\section{Quality control}

Researchers were trained before conducting the study. All measurements were performed by a skilled operator who was blinded to treatments. Scans for each site were repeated three times. The ambient lighting conditions were kept constant during the whole procedure to avoid significant variations in pupil diameter. The right eye of each subject was selected for OCT scanning, while the left eye was used for vergence. All measurement items were sequentially measured in three different accommodation conditions ( $-6.0 \mathrm{D}$ accommodation, relaxation and cycloplegia states). The images of these eyes were independently evaluated by two observers blinded to treatments, as previously reported 

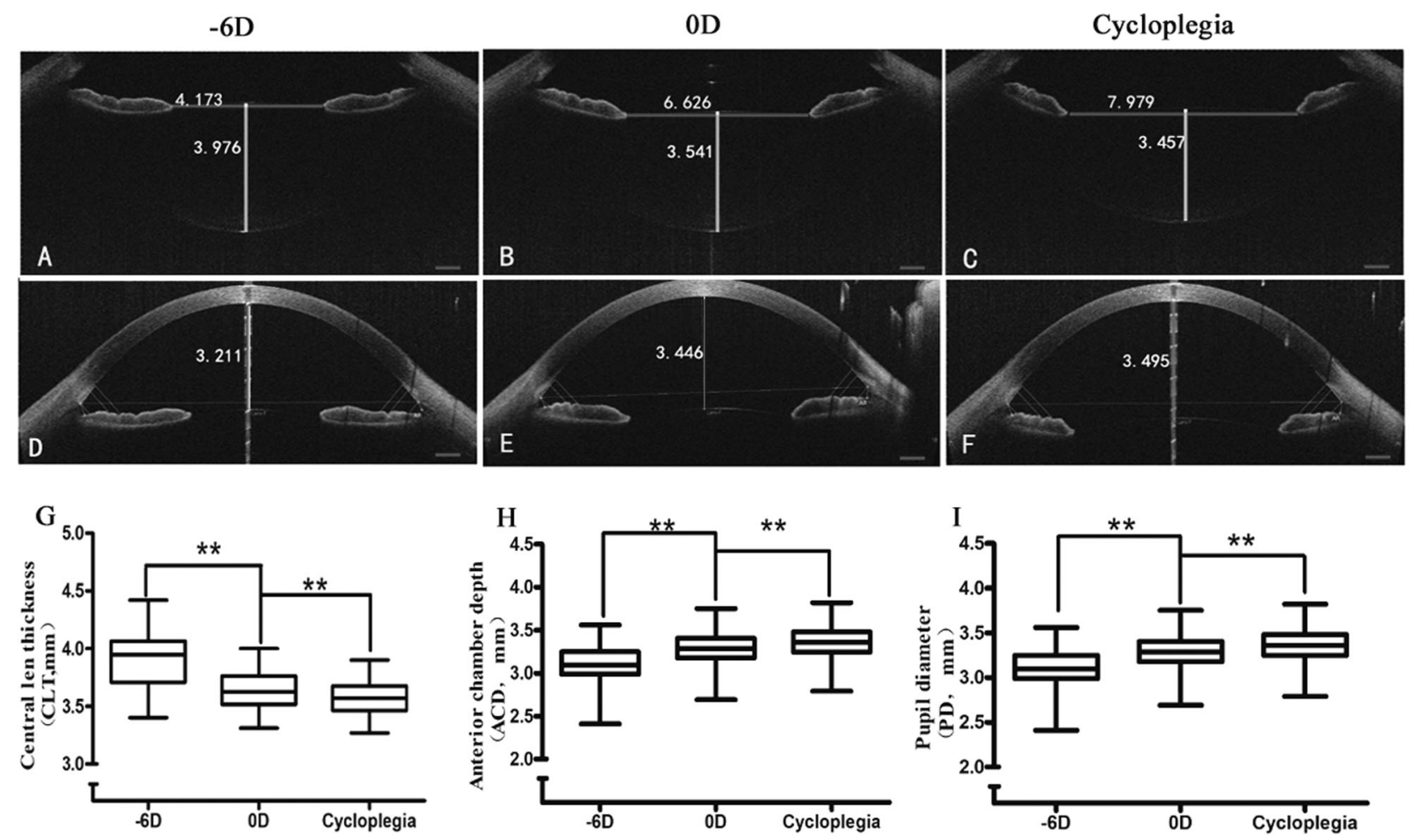

Fig. 2 The central lens thickness (CLT), anterior chamber depth (ACD) and pupil diameter (PD) in different accommodation states of the eye. a, d Examples of CLT, ACD and PD in the -6.0 D accommodation state. $\mathbf{b}$, e Examples of CLT, ACD and PD in the relaxation

[14]. To measure intraobserver repeatability, each image was measured by one observer two separate times with an interval of 3 days, and agreement between the two observations was analysed. To measure interobserver reproducibility, the same images were evaluated by two observers, and the agreement between them was determined. Intraclass correlation coefficients were calculated using a two-way mixed effects model.

\section{Statistical analyses}

Results were evaluated using SPSS software package version19.0 (IBM Corp., Armonk, NY, USA). Sample size estimation was based on the assumption that there was a difference in SCA between different accommodation states. We computed the sample size needed for a repeated measures analysis of variance (rANOVA). A medial level of partial eta square of 0.06 was adopted, which yielded an effect size of about 0.25 . A sample size of at least 28 participants was deemed sufficient for a power of 0.80 with $95 \%$ confidence. The final sample size was adjusted to 34 based on the $20 \%$ participant loss. Quantitative data are presented as mean \pm standard deviation. Repeated measures ANOVA were performed to assess differences among different accommodation states. Prior to repeated measures state. c, f Examples of CLT, ACD and PD in the cycloplegia state. $\mathbf{g}-\mathbf{i}$ Statistical graphs of CLT, ACD and PD in different accommodation states $(* * P<0.01)$

ANOVA, the sphericity assumption was checked using Mauchly's sphericity test. When the sphericity test was not statistically significant, Greenhouse-Geisser correction was applied. Bonferroni post-hoc test was used for comparisons between groups. $P<0.05$ was deemed statistically significant for all cases.

\section{Results}

A total of 34 children aged between 7 and 14 years old were recruited. Four children were excluded due to poor patient cooperation or low quality of OCT images. Thus, a total of 30 patients (16 male; 14 female) were eventually included in the analyses. The mean values for various variables were as follows: age, $12.07 \pm 2.27$ years; best corrected visual acuity, $1.08 \pm 0.12 ; \mathrm{AL}, 24.61 \pm 1.33 \mathrm{~mm}$; refraction, -3.05 \pm 2.53 dioptres; intraocular pressure, $15.24 \pm 2.65 \mathrm{mmHg}$; and amplitude of accommodation, $10.23 \pm 2.12$ dioptres.

\section{Accommodation state changes achieved from accommodation stimulus or cycloplegia}

To determine whether artificial accommodation states were established, we analysed changes in CLT, ACD and PD. 
Significant differences among different accommodation states were observed $\left(F_{\mathrm{CLT}}=112.9, P_{\mathrm{CLT}}=0.00 ; F_{\mathrm{ACD}}=\right.$ $\left.153.8, P_{\mathrm{ACD}}=0.00 ; F_{\mathrm{PD}}=271.4, P_{\mathrm{PD}}=0.00\right)$. When provided with $-6.0 \mathrm{D}$ accommodation stimulation, CLT increased from $3.62 \pm 0.17 \mathrm{~mm}$ to $3.89 \pm 0.24 \mathrm{~mm} \quad(P<$ $0.001)$, whereas ACD decreased from $3.28 \pm 0.23 \mathrm{~mm}$ to $3.09 \pm 0.26 \mathrm{~mm}(P<0.001)$ and PD decreased from $5.71 \pm$ $0.86 \mathrm{~mm}$ to $4.62 \pm 0.73 \mathrm{~mm}(P<0.001)$. Under the state of cycloplegia with tropicamide, CLT decreased from $3.62 \pm$ $0.17 \mathrm{~mm}$ to $3.57 \pm 0.15 \mathrm{~mm}(P<0.001)$, whereas ACD increased from $3.28 \pm 0.23 \mathrm{~mm}$ to $3.35 \pm 0.22 \mathrm{~mm}(P<$ $0.001)$ and PD increased from $5.71 \pm 0.86 \mathrm{~mm}$ to $7.90 \pm$ $0.51 \mathrm{~mm}(P<0.001$; Fig. 2$)$.

\section{Ciliary muscle thickness in different accommodation states of the eye}

The thickness of ciliary body muscles in different accommodation states was analysed. Two sites of ciliary muscles which were $2 \mathrm{~mm}$ and $3 \mathrm{~mm}$ from the scleral spur were tested. Ciliary muscle thickness changed at CM2 $(F=12.9, \quad P=0.00)$ and CM3 $(F=25.0, \quad P=0.00)$. Compared to that in the basal state, ciliary muscle thickness at $2 \mathrm{~mm}$ from the scleral spur increased under the cycloplegia state $(495.8 \pm 68.8 \mu \mathrm{m}$ vs $468 \pm 69 \mu \mathrm{m}, P<$ $0.05)$ but not in response to the accommodation stimulus $(454.3 \pm 63.9 \mu \mathrm{m}$ vs $468.1 \pm 69.3 \mu \mathrm{m}, P>0.05$; Fig. 3). Ciliary muscle thickness at $3 \mathrm{~mm}$ from the scleral spur decreased in response to the accommodation stimulus $(271.0 \pm 7.6 \mu \mathrm{m} \quad$ vs $292.4 \pm 7.8 \mu \mathrm{m}, \quad P<0.05) \quad$ and increased under the cycloplegia state $(309.6 \pm 8.4 \mu \mathrm{m}$ vs $292.4 \pm 7.8 \mu \mathrm{m}, P<0.05$ ).

\section{Schlemm's canal changes in different accommodation states of the eye}

Compared to those in the relaxation state, mean values of SCA $(F=10.959, P<0.05)$, SCL $(F=8.345, P<0.05)$ and SCW $(F=5.107, P<0.05)$ were significantly changed after $-6.0 \mathrm{D}$ accommodation stimulation or cycloplegia with tropicamide was induced. After $-6.0 \mathrm{D}$ accommodation stimulation, on average, SCA increased by $22.80 \%$ (7824 \pm $2727 \mu \mathrm{m}^{2}$ vs $\left.6371 \pm 2517 \mu \mathrm{m}^{2}, P<0.05\right)$, SCL by $18.76 \%$ $(295 \pm 12 \mu \mathrm{m}$ vs $249 \pm 10 \mu \mathrm{m}, P<0.05)$ and SCW by $16.53 \%(31 \pm 8 \mu \mathrm{m}$ vs $27 \pm 9 \mu \mathrm{m}, P<0.05)$.After induction of cycloplegia with $1 \%$ tropicamide, on average, SCA decreased by $21.37 \%\left(5009 \pm 2029 \mu \mathrm{m}^{2}\right.$ vs $6371 \pm$ $\left.2517 \mu \mathrm{m}^{2}, P<0.05\right)$, SCL by $14.76 \% \quad(212 \pm 14 \mu \mathrm{m}$ vs $249 \pm 10 \mu \mathrm{m}, P<0.05)$ and SCW by $17.90 \%(22 \pm 5 \mu \mathrm{m}$ vs $27 \pm 9 \mu \mathrm{m}, P<0.05$; Fig. 4$)$. The changes in SCA $(r=0.35$, $P=0.0007)$, SCL $(r=0.251, P=0.0172)$ and SCW $(r=$ $0.253, P=0.016)$ were significantly correlated with changes in CLT (Fig. 4).
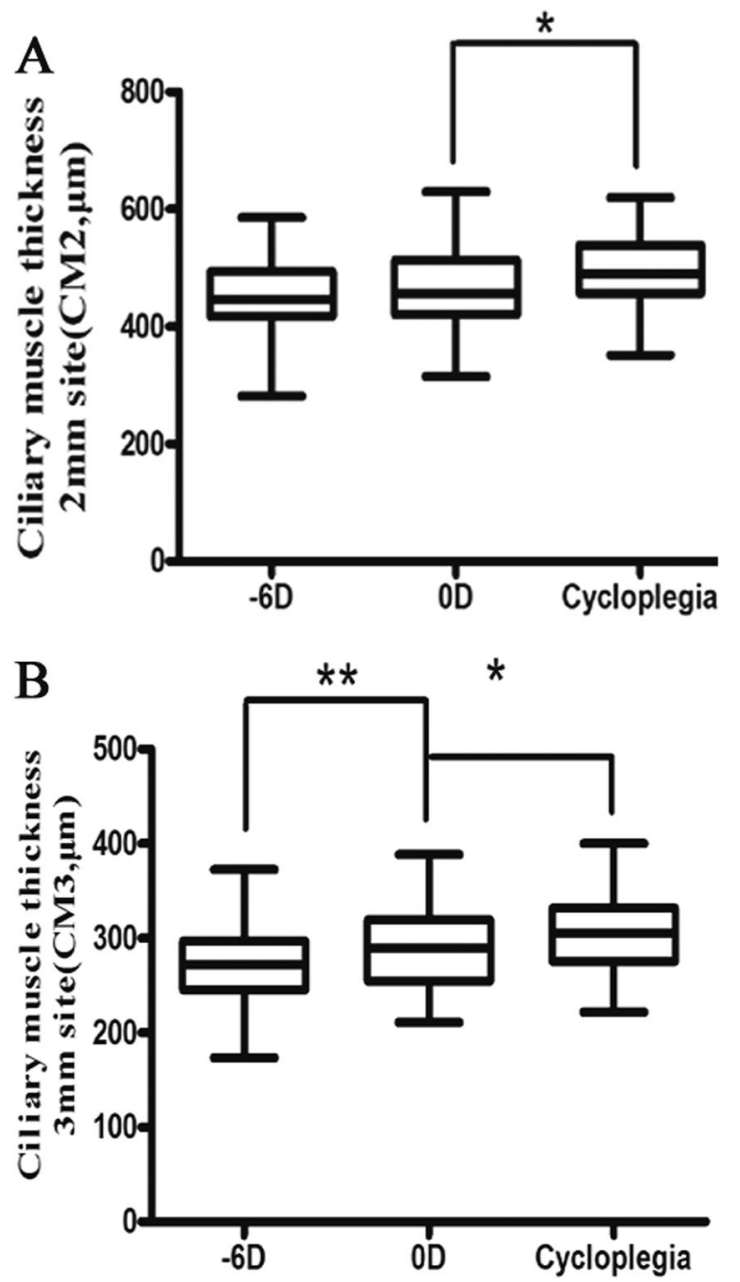

Fig. 3 Ciliary muscle thickness in different accommodation states of the eye. a, b show sites $2 \mathrm{~mm}$ and $3 \mathrm{~mm}$ posterior to the scleral spur, respectively $(* * P<0.01, * P<0.05)$

\section{Trabecular meshwork changes in different accommodation states of the eye}

TM width was not significantly different across different accommodation states $(F=2.48, P=0.92)$. TM length was significantly changed $(F=15.8, P=0.00)$. Compared to that in the basal state, TM length increased by $4.49 \%$ on average $(587 \pm 46 \mu \mathrm{m}$ vs $562 \pm 45 \mu \mathrm{m}, P<0.05)$ after $-6.0 \mathrm{D}$ accommodation stimulation, but it did not change significantly after induction of cycloplegia with tropicamide (Fig. 5).

\section{Discussion}

To our knowledge, this study is the first in vivo study reporting on the effects of different accommodation states on changes in human TM and SC structure in myopia patients. These findings may deepen our understanding of 
Fig. 4 Schlemm's canal (SC) changes in different accommodation states. a, $\mathbf{d}$ Typical optical coherence tomography (OCT) image of SC and trabecular meshwork (TM) in the $-6.0 \mathrm{D}$ accommodation state. b, e Typical OCT image of $\mathrm{SC}$ and TM in the relaxation state. c, $\mathbf{f}$ Typical OCT image of $\mathrm{SC}$ and TM in the cycloplegia state. Scale bar for $\mathbf{a}-\mathbf{c}=$ $500 \mu \mathrm{m}, \mathbf{d}-\mathbf{f}$ depicts large view with scale bar $=200 \mu \mathrm{m})$. $\mathbf{g}-\mathbf{i}$ Statistical graphs of Schlemm's canal area (SCA), Schlemm's canal length (SCL) and Schlemm's canal width (SCW) in different accommodation states $(* * P<0.01)$. J-L: SCA, SCL and SCW changes correlated to changes in central lens thickness (CLT)

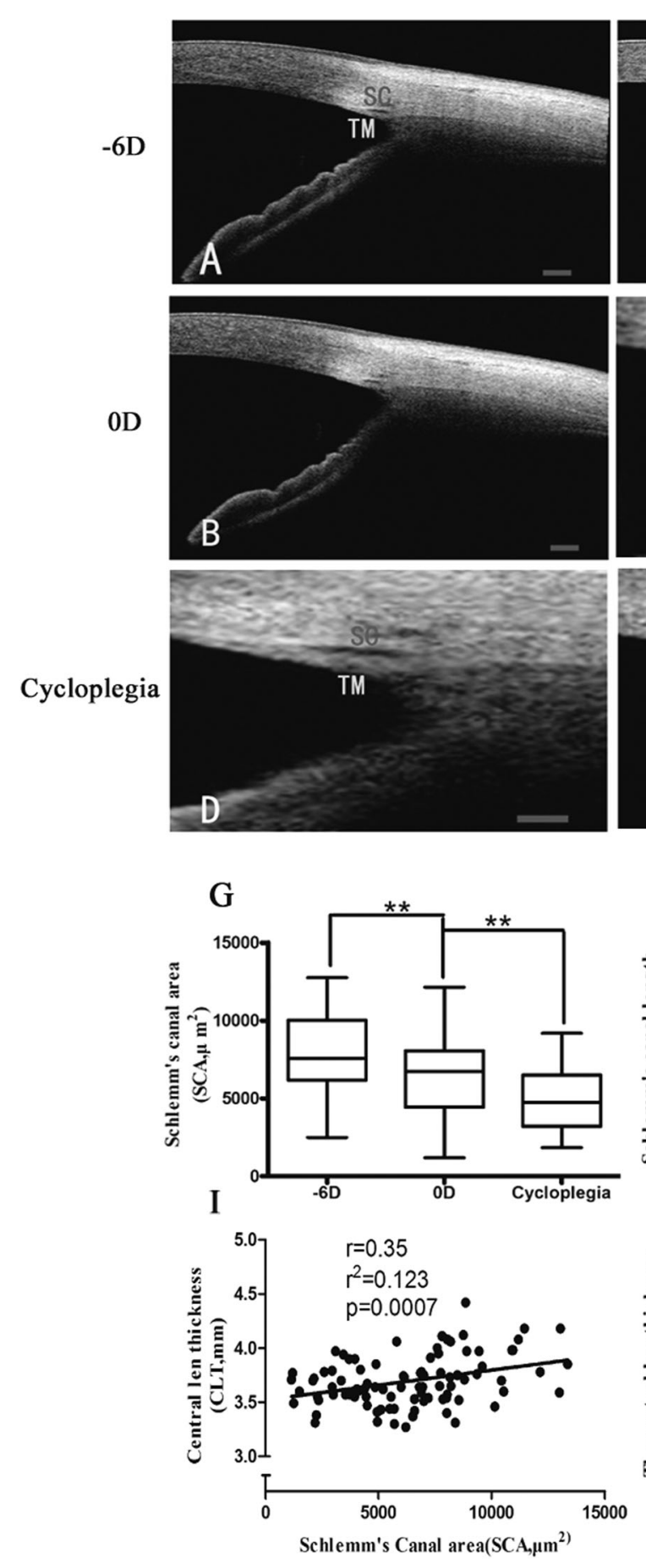

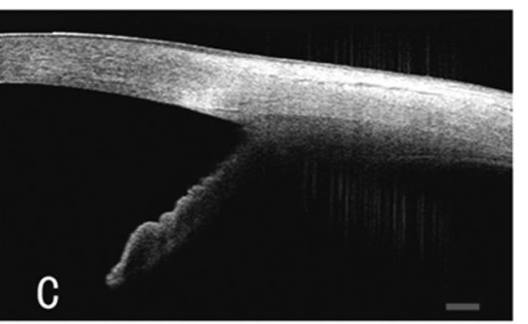
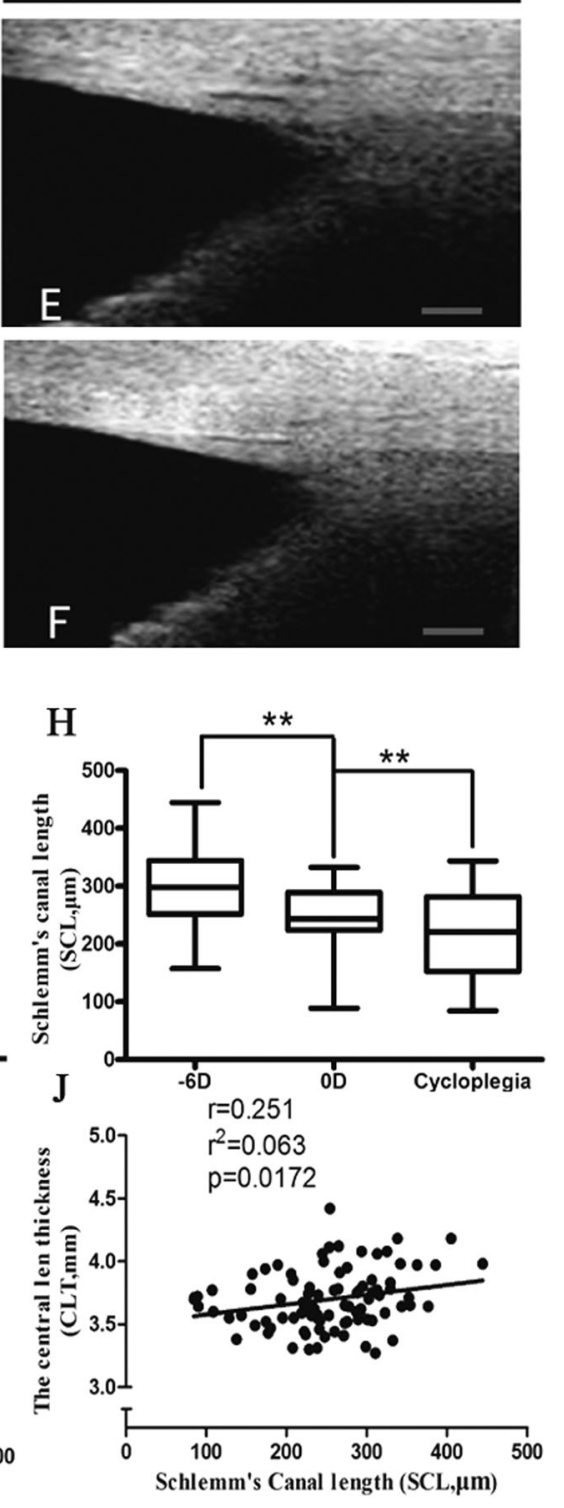

the mechanisms underlying IOP regulation, aqueous humour outflow and pathology of glaucoma.

In the present study, myopia children were recruited as they required cycloplegia for optometry, and there was no additional burden of pharmacological intervention. Two artificial accommodation states were established. Paralysis of accommodation was achieved by tropicamide which is normally used in clinics and is known to be safe when used in children with myopia. Accommodation stimulation was achieved as reported by Ferrer Blasco and colleagues [12]. To verify these two artificial accommodation states, parameters associated with accommodation, including CLT, ACD and PD were tested. CLT increased after $-6.0 \mathrm{D}$ accommodation stimulation but decreased following cycloplegia. In contrast, ACD and PD decreased after $-6.0 \mathrm{D}$ accommodation stimulation but increased after induction of cycloplegia with tropicamide. Thus, two typical artificial accommodation states 
Fig. 5 Trabecular meshwork changes in different accommodation states of the eye. a Statistical graphs of trabecular meshwork width (TMW) in different accommodation states of the eye. b Statistical graphs of trabecular meshwork length (TML) in different accommodation states of the eye $(* * P<0.01)$

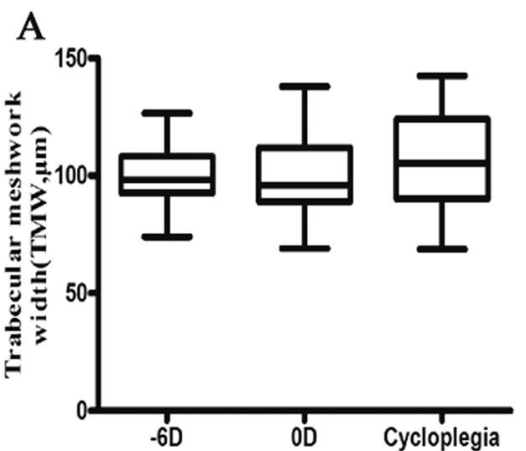

were established in myopia children as previously reported $[12,15,16]$

Ciliary body muscles change with accommodation. Changes in CMs provide direct evidence of accommodation stimulation and cycloplegia. Past research has explored changes in ciliary muscle structure with accommodation using UBM, MRI and OCT [17-21]. The results indicate that changes in shape occurred in the anterior portion of the ciliary muscle with accommodation. Ciliary muscle thickness at $1 \mathrm{~mm}$ posterior to the scleral spur increased with accommodation but thinned at CM2 and CM3. In our study, due to limitations of OCT scan depth, we only analysed changes at CM2 and CM3 and observed that their thickness decreased after $-6.0 \mathrm{D}$ accommodation stimulation, consistent with past results $[17,18]$.

In this study, we observed that SC structure changed significantly under different accommodation states. On average, SC area increased by $22.80 \%$, SC length by $18.76 \%$ and SC width by $16.53 \%$ after $-6.0 \mathrm{D}$ accommodation stimulation. Conversely, after induction of cycloplegia with $1 \%$ tropicamide, on average, SC area decreased by $21.37 \%$, SC length by $14.76 \%$ and SC width by $17.90 \%$. Earlier studies reported that accommodation stimulation or pilocapine decreased aqueous humour outflow resistance and lowered IOP [4, 5, 22]. Paradoxically, cycloplegia increased aqueous humour outflow resistance in monkeys, normal people and POAG patients $[7,8]$. The reason for changes in IOP is unclear. However, we speculate that it could be due to changes in SC structure, mainly the SC inner wall and juxtacanalicular tissue (JCT), which are the major sources of aqueous humour outflow resistance under different accommodation states. The power for accommodation derived from ciliary muscle contraction arises from the longitudinal and circularciliary muscles. The longitudinal ciliary muscle is directly connected to the scleral spur in human eyes $[23,24]$. Thus, when the ciliary muscle contracts during the accommodation state, it can also posteriorly and internally pull the sclera spur, which produces widening of the spaces between the corneoscleral trabecular and distension of the outer and endothelial meshwork, thus increasing the giant vacuoles in the SC [25]. The ciliary muscle tendon has elastic-like fibres known as the cribriform plexus which connect directly to the inner endothelial wall of the SC [26]. Ciliary muscle tone can therefore directly influence the SC inner wall and JCT structure through the fibre system of the cribriform plexus [26, 27].

The TM, another important structure in aqueous humour outflow, did not change significantly in size after -6.0 D accommodation stimulation and cycloplegia. Only TM length increased by $4.49 \%$ on average after $-6.0 \mathrm{D}$ accommodation stimulation. We speculate that TM may possess contractile ability. Wiederholt et al. reported a direct role of TM contractility in aqueous outflow regulation [28]. Researchers have demonstrated that TM is innervated by cholinergic nerve terminals and $\alpha$-smooth muscle actin-positive cells in bovine species, mice and primates [1, 29]. In vitro perfusion of the anterior segments (without ciliary muscles) with a cholinergic agonist (pilocapine) induced TM contraction and decreased outflow facility [30]. However, when pilocapine was applied to the entire eye, outflow facility increased in both mice and primates [1, 31]. This evidence indicates that the TM and ciliary muscle are not connected in the same way. Under accommodation stimulation, the TM could be pulled by the ciliary body. However, contraction of the TM itself could offset the stretching effect of the ciliary muscle on TM.

There are some limitations of this study. First, we only observed changes in SC and TM under -6.0 D accommodation stimulation, but not stepwise accommodation (from $-2.0 \mathrm{D}$ to $-6.0 \mathrm{D}$ ). A more detailed assessment of accommodation states could provide more information for daily life situations such as reading, which usually require 2.0-4.0 D accommodation and are known to decrease IOP. Second, we only tested myopia patients. Whether similar differences occur in healthy people or other cases requires further study. Third, this study was limited to children, who are likely to have more compliant tissues. In adults, the 
effects would likely be smaller, but this requires further confirmation.

In conclusion, SC size increased after $-6.0 \mathrm{D}$ accommodation stimulation and decreased after cycloplegia. For TM, only TM length increased under accommodation stimulation. These findings may explain why IOP decreases after accommodation and provides insight into the pathophysiological mechanisms involved in the regulation of IOP and glaucoma.

\section{Summary}

\section{What was known before}

- Accommodation stimulation promotes aqueous humour outflow facility and decreases intraocular pressure.

\section{What this study adds}

- Schlemm's Canal size enlarges after -6D accommodation stimuli and shrinks under cycloplegia

- However, for trabecular meshwork, only the trabecular meshwork length increase under accommodation stimulus state.

Acknowledgements This work was supported by Natural Science Foundation of China (81770921 to H.Z. and 81470632 to J.W.).

Funding This work was supported by the Natural Science Foundation of China (81770921 to H.Z. and 81470632 to J.W.)

\section{Compliance with ethical standards}

Conflict of interest The authors declare that they have no conflict of interest.

Publisher's note: Springer Nature remains neutral with regard to jurisdictional claims in published maps and institutional affiliations.

\section{References}

1. Overby DR, Bertrand J, Schicht M, Paulsen F, Stamer WD, LutjenDrecoll E. The structure of the trabecular meshwork, its connections to the ciliary muscle, and the effect of pilocarpine on outflow facility in mice. Invest Ophthalmol Vis Sci. 2014;55:3727-36.

2. Grant WM. Clinical measurements of aqueous outflow. Am J Ophthalmol. 1951;34:1603-5.

3. Bull H, von Wolff K, Korber N, Tetz M. Three-year canaloplasty outcomes for the treatment of open-angle glaucoma: European study results. Graefe's Arch Clin Exp Ophthalmol. 2011;249:1537-45.

4. Jenssen F, Krohn J. Effects of static accommodation versus repeated accommodation on intraocular pressure. J Glaucoma. 2012;21:45-8.
5. Read SA, Collins MJ, Becker H, Cutting J, Ross D, Savill AK, et al. Changes in intraocular pressure and ocular pulse amplitude with accommodation. Br J Ophthalmol. 2010;94:332-5.

6. Cassidy L, Delaney Y, Fitzpatrick P, Blake J. Effect of accommodation on intraocular pressure in glaucomatous eyes. Ir J Med Sci. 1998;167:17-8.

7. Barany E, Christensen RE. Cycloplegia and outflow resistance in normal human and monkey eyes and in primary open-angle glaucoma. Arch Ophthalmol. 1967;77:757-60.

8. Velasco Cabrera J, Eiroa Mozos P, Garcia Sanchez J, Bermudez Rodriguez F. Changes in intraocular pressure due to cycloplegia. CLAO J. 1998;24:111-4.

9. Marcus MW, de Vries MM, Junoy Montolio FG, Jansonius NM. Myopia as a risk factor for open-angle glaucoma: a systematic review and meta-analysis. Ophthalmology. 2011;118:1989-94. e1982.

10. Mitchell P, Hourihan F, Sandbach J, Wang JJ. The relationship between glaucoma and myopia: the Blue Mountains Eye Study. Ophthalmology. 1999;106:2010-5.

11. Leon AA, Medrano SM, Rosenfield M. A comparison of the reliability of dynamic retinoscopy and subjective measurements of amplitude of accommodation. Ophthalmic Physiol Opt. 2012;32: 133-41.

12. Esteve-Taboada JJ, Ferrer-Blasco T, Aloy MA, Adsuara JE, Cerdá-Durán $\mathrm{P}$, Mimica $\mathrm{P}$, et al. Ocular anatomic changes for different accommodative demands using swept-source optical coherence tomography: a pilot study. Graefe's Arch Clin Exp Ophthalmol. 2017;255:2399-406.

13. Chen Z, Song Y, Li M, Chen W, Liu S, Cai Z, et al. Schlemm's canal and trabecular meshwork morphology in high myopia. Ophthalmic Physiol Opt. 2018;38:266-72.

14. Chen Z, Sun J, Li M, Liu S, Chen L, Jing S, et al. Effect of age on the morphologies of the human Schlemm's canal and trabecular meshwork measured with sweptsource optical coherence tomography. Eye. 2018;32:1621-8.

15. Richdale K, Sinnott LT, Bullimore MA, Wassenaar PA, Schmalbrock P, Kao CY, et al. Quantification of age-related and per diopter accommodative changes of the lens and ciliary muscle in the emmetropic human eye. Invest Ophthalmol Vis Sci. 2013;54:1095-105.

16. Farouk MM, Naito T, Shinomiya K, Eguchi H, Sayed KM, Nagasawa T, et al. Optical coherence tomography reveals new insights into the accommodation mechanism. J Ophthalmol. 2015;2015:510459.

17. Esteve-Taboada JJ, Dominguez-Vicent A, Monsalvez-Romin D, Del Aguila-Carrasco AJ, Montes-Mico R. Non-invasive measurements of the dynamic changes in the ciliary muscle, crystalline lens morphology, and anterior chamber during accommodation with a high-resolution OCT. Graefe's Arch Clin Exp Ophthalmol. 2017;255:1385-94.

18. Lewis HA, Kao CY, Sinnott LT, Bailey MD. Changes in ciliary muscle thickness during accommodation in children. Optom Vis Sci. 2012;89:727-37.

19. Lossing LA, Sinnott LT, Kao C-Y, Richdale K, Bailey MD. Measuring changes in ciliary muscle thickness with accommodation in young adults. Optom Vis Sci. 2012;89:719-26.

20. Stachs O, Martin H, Kirchhoff A, Stave J, Terwee T, Guthoff R. Monitoring accommodative ciliary muscle function using threedimensional ultrasound. Graefe's Arch Clin Exp Ophthalmol. 2002;240:906-12.

21. Sheppard AL, Davies LN. In vivo analysis of ciliary muscle morphologic changes with accommodation and axial ametropia. Invest Opthalmol Vis Sci. 2010;51:6882.

22. YD LCassidy, Fitzpatrick P, Blake. J. Effect of accommodation on intraocur pressure in Glaucomatous Eyes. Ir J Med Sci. 1998;167: $17-9$. 
23. Rohen J. Über den Ansatz der Ciliarmuskulatur im Bereich des Kammerwinkels. Ophthalmologica. 1956;131:51-60.

24. Kupfer C. Relationship of ciliary body meridional muscle and corneoscleral trabecular meshwork. Arch Ophthalmol. 1962;68: 818-22.

25. Grierson I, Lee WR, Abraham S. Effects of pilocarpine on the morphology of the human outflow apparatus. Br J Ophthalmol. 1978;62:302-13.

26. Rohen JW, Futa R, Lutjen-Drecoll E. The fine structure of the cribriform meshwork in normal and glaucomatous eyes as seen in tangential sections. Invest Ophthalmol Vis Sci. 1981;21:574-85.

27. Hann CR, Fautsch MP. The elastin fiber system between and adjacent to collector channels in the human juxtacanalicular tissue. Invest Ophthalmol Vis Sci. 2011;52:45-50.
28. Lepple-Wienhues A, Stahl F, Wiederholt M. Differential smooth muscle-like contractile properties of trabecular meshwork and ciliary muscle. Exp Eye Res. 1991;53:33-8.

29. de Kater AW, Shahsafaei A, Epstein DL. Localization of smooth muscle and nonmuscle actin isoforms in the human aqueous outflow pathway. Invest Ophthalmol Vis Sci. 1992;33:424-9.

30. Wiederholt M, Bielka S, Schweig F, Lutjen-Drecoll E, LeppleWienhues A. Regulation of outflow rate and resistance in the perfused anterior segment of the bovine eye. Exp Eye Res. 1995; 61:223-34.

31. Kaufman PL, Barany EH. Loss of acute pilocarpine effect on outflow facility following surgical disinsertion and retrodisplacement of the ciliary muscle from the scleral spur in the cynomolgus monkey. Invest Ophthalmol. 1976;15:793-807. 